\title{
Acompanhamento a médio prazo da reconstrução acetabular com enxerto ósseo liofilizado bovino e dispositivo de reforço
}

\author{
Mid-term follow-up of acetabular reconstruction using bovine freeze-dried bone \\ graft and reinforcement device
}

\author{
Ricardo Rosito; Carlos Roberto Galia ; Carlos Alberto de Souza Macedo'; Lourdes Maria Araúuo Camargo Quaresma3; \\ LuIs Fernando Moreira, TCBC-RS 4
}

RES U M O

\begin{abstract}
Objetivo: Relatar a capacidade clínica e radiográfica de integração de enxertos ósseos liofilizados bovinos. Método: Vinte e cinco pacientes foram incluídos. O período médio de acompanhamento foi de oito anos. Os enxertos foram purificados e liofilizados. A análise clínica baseou-se no escore de Merle d'Aubigné e Postel, e critérios de pontuação estabelecidos para a osteointegração radiográfica foram usados para as análises radiográficas. Resultados: Bons resultados clínicos e radiográficos foram observados em $80 \%$ e $72 \%$ dos casos, respectivamente. Conclusão: Enxertos liofilizados bovinos podem ser usados com segurança e adequadamente na revisão acetabular da artroplastia total de quadril.
\end{abstract}

Descritores: Enxertos ósseos. Xenoenxertos. Osso bovino.

\section{INTRODUÇÃO}

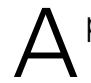
perda óssea acetabular é um dos principais problemas na revisão da artroplastia total de quadril'. Demonstrou-se que o tratamento das deficiências tipo I e II' com osso esponjoso picado impactado oferece bons resultados². Portanto, o tratameto de defeitos graves, tipos III e IV, é um desafio maior. Uma opção valiosa nesses casos é a utilização de aloenxertos em bloco, mas foi demonstrado que o índice de falha de enxertos estruturais, sem o suporte de um dispositivo de reforço, tem aumentado nos últimos $\operatorname{anos}^{3}$. Como alternativa, alguns autores têm defendido, em vez disso, o uso de um anel de reforço acetabular. Esse dispositivo parece proteger os enxertos de estresse excessivo, auxiliando a acomodar o acetábulo reconstruído até que o enxerto esteja integrado ${ }^{4,5}$.

Entretanto, escolher a técnica de reconstrução para um defeito acetabular não é a única peocupação. $O$ uso de enxertos ósseos também é essencial, e enxertos autologos são em pequena quantidade para substituir essas perdas. Além disso, as demandas de tecidos são muito maiores do que a disponibilidade real quando se considera o uso de aloenxertos ${ }^{6}$. Essa situação nos levou a buscar um processamento alternativo de tecidos para submeter os enxertos a algum método de desinfecção e esterilização, bem como tentar usar xenoenxertos de origem bovina. Por essa razão, foi desenvolvido um processo de liofilização; e depois de confirmado inicialmente por estudos experimentais em animais ${ }^{7}$ e seguido pelo uso em outros procedimentos ortopédicos gerais, esta coorte teve início.

Portanto, o objetivo deste estudo foi demonstrar a capacidade de osteointegração clínica e radiográfica de enxertos ósseos bovinos liofilizados, produzidos no nosso Banco de Tecidos no Hospital Universitário do Hospital de Clínicas de Porto Alegre (HCPA, TBHCPA), em procedimentos de reconstrução acetabular.

\section{MÉTODOS}

De maio de 1997 a fevereiro de 2005, 25 pacientes com defeitos acetabulares graves, tipos III e IV da classificação de D'Antonio et al. ${ }^{1}$, foram consecutivamente submetidos a reconstrução acetabular na revisão de artroplastia total de quadril (RTHA), pela mesma equipe de cirurgia de quadril do Departamento de Ortopedia do Hospital Universitário do HCPA. Todos os pacientes foram plenamente informados a respeito do estudo, o qual foi aprovado pelo Comitê de Ética do HCPA, e foi obtido o consentimento livre e esclarecido por escrito. Os pacientes foram acompanhados até janeiro de 2009. Os enxertos eram de oigem bovina. O osso bovino é obtido de ossos de gado brasileiro, e considera-se que esteja completamente livre de Encefalopatia Espongiforme Bovina (EEB). Todos os en-

\footnotetext{
Trabalho realizado no Departamento de Ortopedia - Banco de Tecidos, Hospital de Clínicas de Porto Alegre, Faculdade de Medicina, Universidade Federal do Rio Grande do Sul (UFRGS), Porto Alegre, RS, Brasil.

1. Cirurgião, Departamento de Ortopedia - Banco de Tecidos, Hospital de Clínicas de Porto Alegre, Faculdade de Medicina (UFRGS), Porto Alegre, RS, Brasil; 2. Professor Assistente de Cirurgia, Faculdade de Medicina, UFGRS, Porto Alegre, RS, Brasil; 3. Professor de Cirurgia, Programa de Pós-Graduação em Cirurgia, Faculdade de Medicina, UFGRS, Porto Alegre, RS, Brasil; 4. Biólogo - Empresa Brasileira de Pesquisa Agropecuária (EMBRAPA)
} 
xertos ósseos foram processados no TBHCPA seguindo o nosso próprio protocolo de processamento. Esse processo possibiitou a produção de enxertos ósseos mantendo as principais características (proteínas e minerais) quase inalteradas (Figura 1). O método de preparação não pôde ser totalmente revelado a fim de proteger a propriedade intelectual. No processo, a desnaturação de proteínas com peróxido de hidrogênio a $20 \%$ é seguida pela extração alcoólica de lipídios. O produto final compõe-se de minerais (65\%) e proteínas (27\%). É esterilizado definitivamente em autoclave. A falha da artroplastia foi determinada como asséptica em todos os pacientes. O tipo e extensão dos defeitos acetabulares haviam sido determinados a partir de radiografias pré-operatórias e avaliações intraoperatórias. As características clinicopatológicas dos pacientes são mostradas na tabela 1.

Todos os pacientes foram operados pela mesma equipe e mesma técnica reconstrutiva. Foi realizada abordagem posterior em todos os casos.

\section{Técnica}

A prótese frouxa foi removida e todo cimento, debris, granulomas e membrana fibrosa foram completamen-

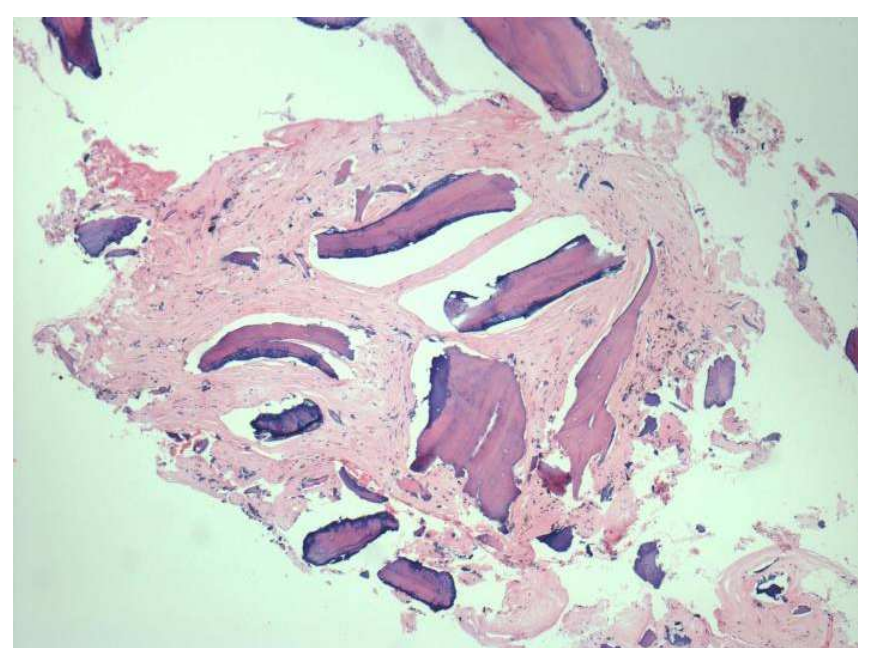

Figura 1 - Amostra histológica de um enxerto bovino com dois anos de acompanhamentomostrando áreas de neoformação óssea e espículas residuais do material de enxerto.

Tabela 1 - Características clinicopatológicas dos pacientes.

\begin{tabular}{lcl}
\hline Características & $\begin{array}{c}\text { Enxerto Bovino } \\
\mathrm{n}=\end{array}$ & $\mathbf{2 5}(\%)$ \\
\hline Mulher & 20 & $(80,0)$ \\
Homem & 5 & $(20,0)$ \\
Idade média (anos) (dp) & 57,7 & $(12,3)$ \\
Acompanhamento médio (meses) (dp) & 96,1 & $(24,49)$ \\
Deficiência acetabular & & \\
Tipo III & 14 & $(56)$ \\
Tipo IV & 11 & $(44)$ \\
\hline
\end{tabular}

$d p=$ desvio-padrão. te removidos. Foi realizada cuidadosa fresagem da cavidade do acetábulo a fim de alcançar o leito ósseo vascular, e a seguir o acetábulo foi reconstruído com osso esponjoso liofilizado picado (tamanho dos fragmentos, aproximadamente $8 \mathrm{~mm}^{3}$ ). Os fragmentos foram comprimidos nos defeitos do acetábulo e cuidadosamente condensados. As flanges do dispositivo de reforço (MDTOे, São Paulo, Brasil) foram curvadas no formato necessário para se ajustar à anatomia específica da região acetabular. O gancho do dispositivo foi colocado sob a porção em forma de gota de lágrima. A flange superior do anel de metal foi parafusada ao ílio. Isso deve resultar numa composição estável (consistindo no osso hospedeiro que suporta o peso, enxertos e implante) com um enxerto ósseo impactado localizado abaixo do anel. Em seguida, uma cúpula de polietileno foi cimentada no dispositivo de reforço acetabular. A quantidade de enxerto ósseo utilizada variou de $40 \mathrm{~g}$ a $60 \mathrm{~g}$ em todos os casos.

A análise dos pacientes baseou-se na avaliação clínica e radiográfica. A análise clínica baseou-se em critérios funcionais estabelecidos por Merle d'Aubigné e Postel ${ }^{8}$.

Para as análises radiográficas, foram usadas várias características subjetivamente estabelecidas, tais como radioluscência, densidade, formação óssea trabecular e migração de componentes. Assim, foi realizada uma análise radiográfica da osteointegração baseada nos critérios de Conn et al. ${ }^{9}$ para estabelecer a incorporação óssea dos enxertos nos dois grupos. Cada critério, exceto a migração, recebeu uma pontuação independente variando de 0 a 2 para cada uma das três zonas de De Lee and Chanrley ${ }^{10}$ no acetábulo, em que 0 era o pior resultado e 2 , o melhor ${ }^{11}$. A soma das pontuações foi, então, multiplicada por três para o acetábulo. Para a migração, uma pontuação de 2, 4 ou 6 foi estabelecida quando havia mais do que $6 \mathrm{~mm}, 3$ a 5 $\mathrm{mm}$, ou menos de $3 \mathrm{~mm}$ de deslocamento da prótese. Portanto, um total de 24 pontos poderia ser obtido para a avaliação acetabular. Foram considerados adequados os resultados com uma soma de 19 ou mais.

\section{RESULTADOS}

Nenhuma complicação grave ocorreu no período pós-operatório inicial. Apenas duas mortes, dois e seis anos após o procedimento foram registradas, não relacionadas com a RTHA. Nenhum outro paciente foi perdido para o acompanhamento.

No geral, clinicamente não foram observadas intercorrências. Entretanto, um caso de infecção superficial (celulite) ocorreu seis meses depois do procedimento e foi tratado com sucesso através de antibióticos. Resultados clínicos e radiográficos (Figuras 2-4) são mostrados nas tabelas 2 e 3 , respectivamente.

\section{DISCUSSÃO}

Os objetivos da reconstrução de defeitos acetabulares graves na revisão da artroplastia de quadril 


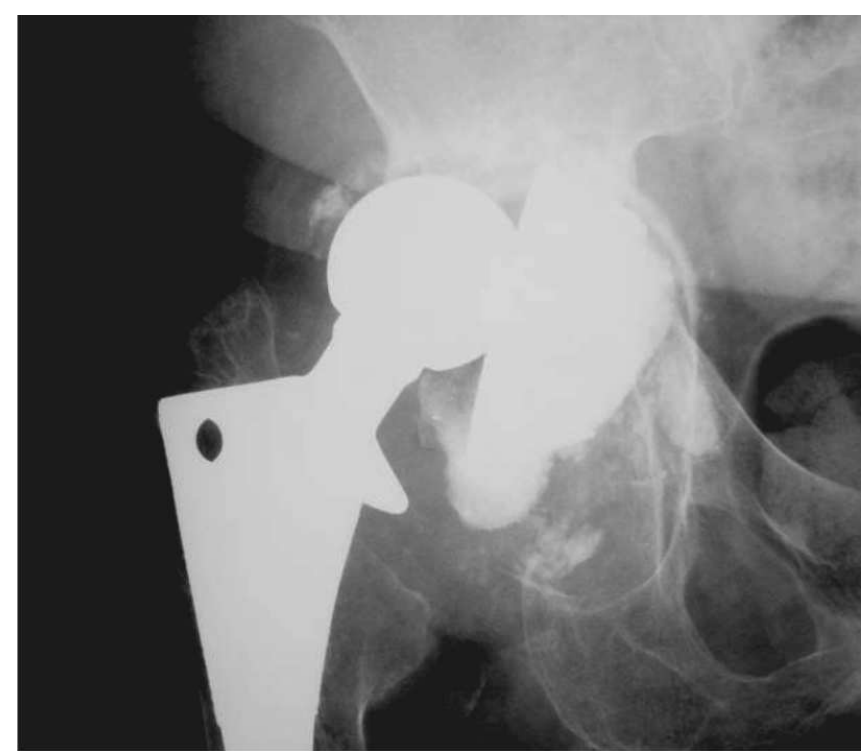

Figura 2 - Radiografia anteroposterior do afrouxamento acetabular, deficiência tipo IV segundo D'Antonio et al. ${ }^{\prime}$

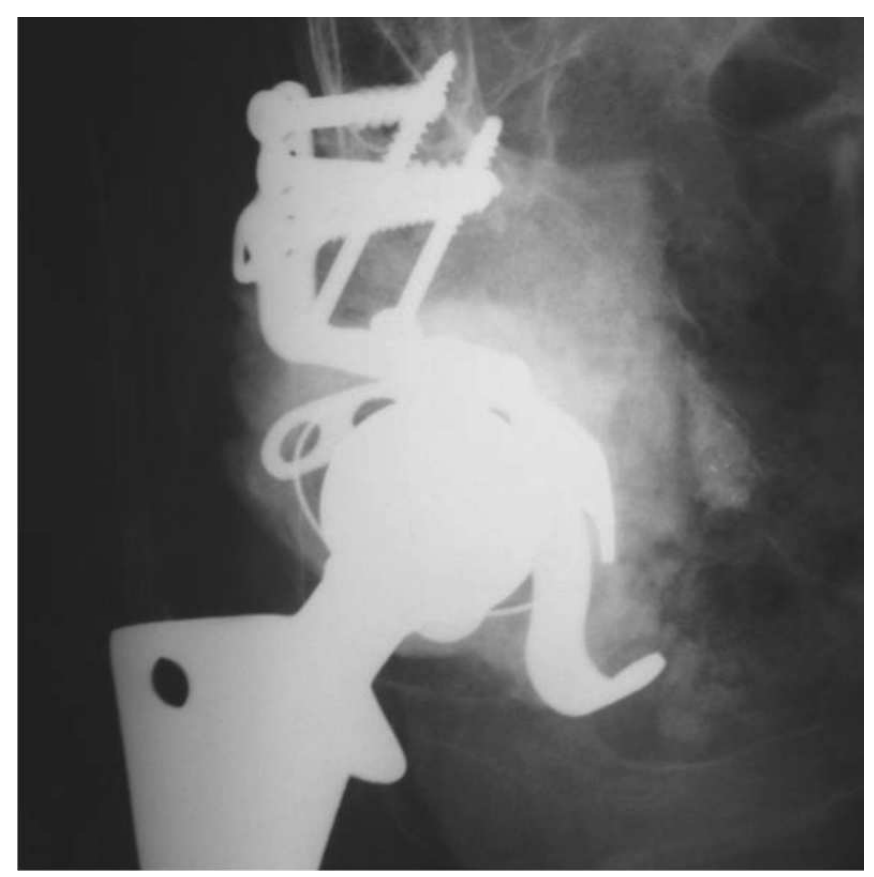

Figura 3 - Radiografia no pós-operatório imediato mostrando a reconstrução acetabular com enxerto bovino liofilizado e dispositivo de reforço.

são restaurar o estoque ósseo, reparar a mecânica do quadril e obter estabilidade, e o uso de enxerto ósseo é imperativo para alcançá-los. Autoenxertos são excelentes, mas sua quantidade geralmente é limitada, e assim os aloenxertos têm sido frequentemente utilizados. Portanto, um banco de ossos sob estrito controle de qualidade é necessário para minimizar o risco de transmissão de doença. Entretanto, não há uma técnica única que forneça uma solução para todas as deficiências. A reconstrução com osso esponjoso picado impactado tem dado bons resultados ${ }^{2,12}$, mas há certo ceticismo a respeito de seu uso em acetábulos com complicações, especialmente aqueles nas deficiências

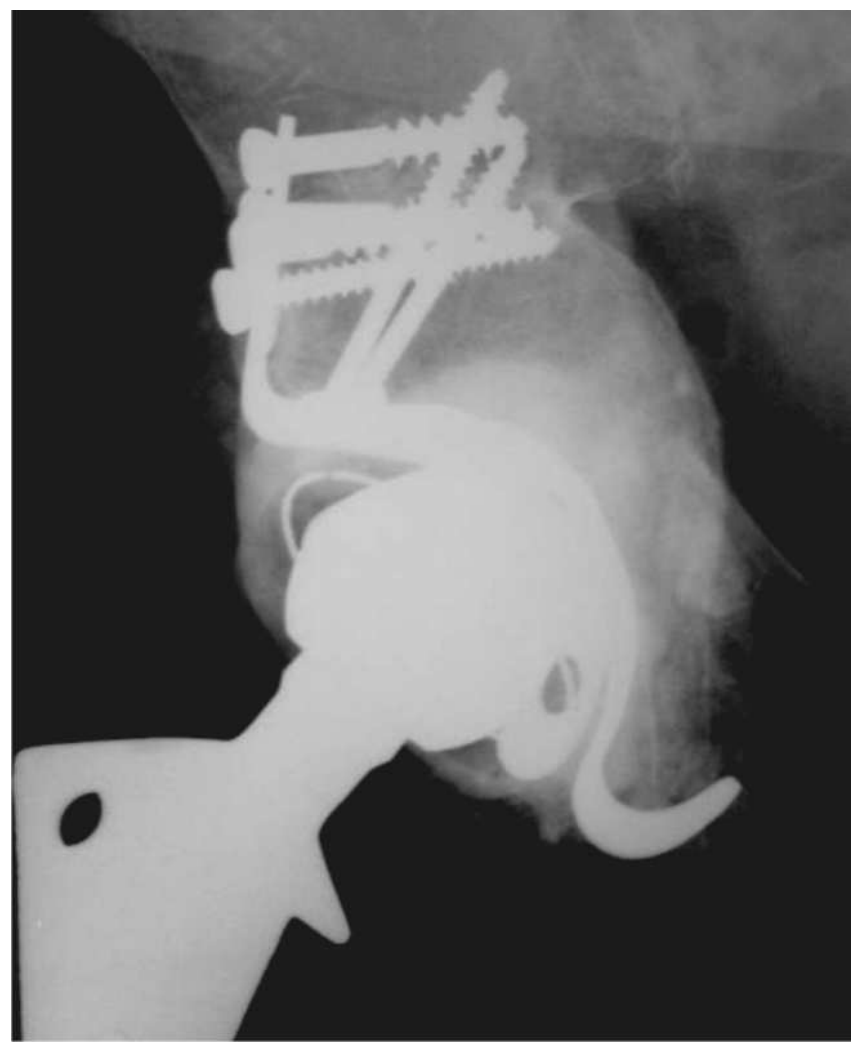

Figura 4 - Radiografia anteroposterior da reconstrução com enxerto bovino liofilizado e dispositivo de reforço (acompanhamento de 3 anos), mostrando sinais sugestivos de incorporação óssea, conforme se pode ver pela regularidade da parede média, a densidade do enxerto e a ausência de linhas radiolucentes.

de tipos III e IV ${ }^{1,4,5,13}$. Além disso, a reconstrução de aloenxertos estruturais ainda é controversa ${ }^{3}$. Assim como outros, estes autores também consideram que a ocorrência de um defeito acetabular grave é uma indicação para a reconstrução através de dispositivo de reforço combinado com enxertos ósseos. Esse método provê estabilidade inicial, protege os enxertos de estresse mecânico até que a integração do enxerto seja alcançada ${ }^{4,5}$.

Para o procedimento de quadril, acredita-se que o enxerto mais adequado seria aquele que tivesse sido ligeiramente modificado por algum processo. Nas análises físicas e químicas, os ossos liofilizados produzidos no TBHCPA mantiveram a maioria das características dos minerais e proteínas virtualmente inalteradas, e os enxertos de fontes bovina e humana são bastante semelhantes, embora não mantenham a mesma textura e maleabilidade que equivalentes congelados ${ }^{14}$. Isso permitiu o manuseio adequado dos ossos liofilizados, tanto do ponto técnico quanto mecânico, após a reidratação ${ }^{15}$.

Pelos critérios de Merle d'Aubigné e Postel ${ }^{8}$, os resultados médios obtidos para enxertos bovinos foram considerados bons e muito bons em $80 \%$ dos casos. Embora o acompanhamento possa ser considerado não suficientemente longo para uma avaliação clínica mais confiável, é indicativo de que o uso dos enxertos liofilizados bovinos durante esse período não causou qualquer prejuízo aos 
Tabela 2 - Resultados clínicos.

\begin{tabular}{|c|c|}
\hline $\begin{array}{l}\text { Avaliação Clínica } \\
\text { Merle d'Aubigné e Postel }\end{array}$ & $\begin{array}{c}\text { Enxerto Bovino } \\
(\mathrm{n}=25) \mathrm{n}(\%)\end{array}$ \\
\hline \multicolumn{2}{|l|}{ Muito bom e bom } \\
\hline 12 & $8(20)$ \\
\hline 11 & 9 (35) \\
\hline 10 & $2(12)$ \\
\hline Total & $19(76)$ \\
\hline \multicolumn{2}{|l|}{ Médio, razoável e ruim } \\
\hline 9 & $3(12)$ \\
\hline 8 & $1 \quad(4)$ \\
\hline 7 or $<7$ & $2 \quad(8)$ \\
\hline Total & $6(24)$ \\
\hline
\end{tabular}

Tabela 3 - Resultados das radiografias.

\begin{tabular}{lr}
\hline $\begin{array}{l}\text { Pontuações da avaliação } \\
\text { das radiografias (\%) }\end{array}$ & $\begin{array}{r}\text { Enxerto Bovino } \\
(n=25)\end{array}$ \\
\hline $\begin{array}{l}\text { Muito bom e bom } \\
24-22\end{array}$ & $15(60)$ \\
$21-19$ & $3(12)$ \\
Total & $18(72)$ \\
Médio, razoável e ruim & 2 \\
$18-16$ & $2(8)$ \\
$15-13$ & $3(12)$ \\
$<13$ & $7(28)$ \\
Total &
\end{tabular}

pacientes ou diferenças significativas entre eles. Comparando-se esses resultados com os da literatura para período de acompanhamento semelhante - porém com a utilização de enxertos alogênicos congelados -, não se observaram diferenças consideráveis que pudessem ser atribuídas ao uso de enxertos bovinos liofilizados $4,5,13$.

Vários estudos avaliaram clínica e radiograficamente, em diversas doenças ósseas, o uso de enxertos humanos e bovinos liofilizados, demonstrando resultados muito bons. Entretanto, poucos artigos indexados a respeito do uso de enxertos humanos e bovinos liofilizados na RTHA foram relatados. Essa relutância dos cirurgiões de quadril em usar enxertos liofilizados pode estar ralacionada, em parte, com o número de enxertos disponíveis com diferentes etapas no processo de produção para propósitos e indicações distintas também. Consequentemente, diferentes respostas mecânicas e biológicas podem ser obtidas, levando a uma preocupação injustificada quanto ao uso desse tipo de enxerto ${ }^{16}$. Levai e Boisgard ${ }^{14}$ reportaram trinta casos de revisões realizadas em substitições totais de quadril por afrouxamento com uma técnica específica para reconstrução acetabular, combinando o uso de um substituto de osso bovino e um anel de suporte. Não se observou migração do implante acetabular ou osteólise do heteroenxerto em 27 (90\%) dos casos em três anos após o procedimento. Radiologicamente, o heteroenxerto gradualmente se condensou, e sua aparência era semehante à observada com aloenxertos. Nessa série, as duas falhas com migração de implante e ostreólise de heteroenxerto foram consideradas viés técnico associado ao uso do anel de Muller, e em ambos os casos, o suporte era dado pelo heteroenxerto esponjoso e não pelo osso hospedeiro ${ }^{14}$. De Roeck e Drabu ${ }^{17}$ relataram sobre 32 pacientes que tiveram RTHA utilizando componentes cimentados e aloenxerto impactado de ossos liofilizados processados. A resistência global com esse tipo de enxerto num acompanhamento médio de quatro anos foi de $91 \%$. Não houve falha dos componentes femorais, embora revisão tivesse sido necessária em três pacientes devido a falha no acetábulo. O enxerto liofilizado pode requerer reidratação mais prolongada para impactação adequada. Os resultados do enxerto ósseo apenas com impactação com osso liofilizado têm sido satisfatórios, embora esses autores não se sintam inteiramente seguros com seu uso isolado nos casos de acetábulo com complicações ${ }^{17}$. Thien et al. ${ }^{18}$ relataram um índice geral de sobrevida para reconstruções acetabulares de $86 \%$ em sete casos em que foram utilizados fragmentos de ossos esponjosos liofilizados impactados e uma cúpula cimentada, com uma média de acompanhamento de sete anos (variação, 5-9 anos). Nesse período mediano de acompanhamento, não foi observado afrouxamenrto asséptico e os resultados para aloenxerto com fragmentos de osso liofilizado foram aceitáveis. Charalambides et al. ${ }^{19}$ publicaram um trabalho enfocando os resultados ruins em 27 casos de RTHA, acompanhados por uma média de 2,5 anos, após a utilização de autoenxerto e xenoenxerto (Surgibone) combinados. Dezessete (62\%) dentre os 27 pacientes mostraram incorporação óssea aparente dentro de três meses. Em três (11\%) pacientes, no entanto, não houve incorporação. Três outros casos (11\%) pareceram apresentar o que eles consideraram uma pseudoinfecção (sem agente identificado); e um paciente, que teve o procedimento de revisão novamente revisto, sofreu infecção profunda por MRSA. Desconsiderando o caso de infecção inequívoca, não relacionada ao enxerto, e mesmo considerando os três casos de pseudoinfecção, que podem não ter relação com os enxertos, encontra-se $77 \%$ de resultados bons, o que é semelhante aos observados em outros métodos, e, portanto, seus resultados ruins são discutíveis. Além disso, os autores também mostraram uma amostra histológica daquele paciente que exigiu, apesar da aparente incorporação radiográfica, revisão da substituição devido a afrouxamento acetabular. Foram obseradas neoformação óssea a partir da área enxertada e espículas ósseas necróticas residuais do material de enxerto, o que demonstra claramente a incorporação do enxerto ${ }^{20}$.

Consideriando-se os critérios radiográficos, a despeito de vieses, os resultados obtidos com enxertos ósseos bovinos liofilizados do TBHCPA, nesta série, foram comparáveis entre si e àqueles relatados na literatura, mesmo aqueles relatos do uso de aloenxertos congelados. Utiizando uma técnica semelhante de impactação em enxerto congelado e cimento, e com acompanhamento similar, Kerboull 
et al. ${ }^{4}$ reportaram um índice semelhante, de $92 \%$ de incorporação de enxerto. Portanto, as realizações obtidas na RTHA parecem estar mais relacionados à habilidade do cirurgião, limitações inerentes às técnicas e à gravidade do caso, e não ao tipo de enxerto usado.

O uso de enxertos ósseos liofilizados provê uma redução no risco de transmissão de doenças infcciosas e tumores, uma vez que são usados reagentes químicos no seu processamenro para inativar bactérias, vírus e, provavelmente, príons, devido à exposição ao hipoclorito de sódio ${ }^{21}$. Depois do processo todo, o osso é também submetido a algum tipo de esterilização ${ }^{22,23}$ que em nosso banco de tecidos atinge virtualmente $100 \%$ de eficácia. Dessa forma, as preocupações relacionadas com a transmissão de príons (EEB), atribuída ao uso de enxerto ósseo bovino liofilizado parecem inadequadas. Além disso, a seleção cuidadosa e o país de origem do rebanho, especialmente o Brasil, que tem sido sempre um país livre de risco para EEB, devem ser considerados ${ }^{24}$.
Do ponto de vista mecânico, alguns estudos em que se utiliza osso liofilizado não-descalcificado concluiram que não há diferença mecânica entre osso liofilizado e osso congelado, e se há alguma, é em favor dos liofilizados, uma vez que eles não possuem gordura, sangue e células de medula15,25.

Embora sejam escassos os dados a respeito do uso de xenoenxertos na RTHA, não foram observadas complicações clínicas, exceto aquelas esperadas com o uso de aloenxertos ou xenoenxertos em geral, uma vez que perfis físico-químicos confirmaram que os ossos são semelhantes $^{26,27}$. Os resultados obtidos, portanto, demonstraram que o uso de enxertos ósseos liofilizados bovinos não traz reações adversas de qualquer tipo, reforçando ainda mais a sua segurança.

Pode-se concluir que o processo de liofilização de ossos de origem bovina, realizado em banco de tecidos, tem qualidade adequada para ser usado na RTHA.

\title{
A B S T R A C T
}

\begin{abstract}
Objective: To report clinical and radiographic graft incorporation capability of bovine freeze-dried bone grafts. Methods: Twenty five patients were enrolled. The mean follow-up was eight years. Grafts were purified and freeze-dried. Clinical analysis was based on the score of Merle d'Aubigné and Postel and an established score criteria for radiographic bone incorporation was used for radiographic analyses. Results: Good clinical and radiographic results were found in $80 \%$ and $72 \%$ of the cases, respectively. Conclusion: Bovine freeze-dried grafts can be safely and adequately used in acetabular revision in total hip arthroplasty.
\end{abstract}

Key words: Bone grafts. Xenografts. Bovine bone.

\section{REFERENCIAS}

1. D'Antonio JA, Capello WN, Borden LS, Bargar WL, Bierbaun BF, Boettcher WG, et al. Classification and management of acetabular abnormalities in total hip arthroplasty. Clin Orthop. 1989; 243:12637.

2. Schreurs BW, Slooff TJ, Buma P, Gardeniers J, Huiskes R. Acetabular reconstruction with impacted morsellised cancelous bone graft and cement. A10 to 15 year follow-up study of 60 revision arthroplasties. J Bone Joint Surg Br. 1998; 80B: 391-5.

3. Shinar AA, Harris WH. Bulk structural autogenous grafts and allografts for reconstruction of the acetabulum in total hip arthroplasty. Sixteen-year-average-follow-up. J Bone Joint Surg Am.1997; 79A:159-68.

4. Kerboull M. Hamadouche M, Kerboull L. The Kerboull acetabular reinforcement device in major acetabular reconstructions. Clin Orthop. 2000; 378:155-68.

5. Winter E. Piert M. Volkmann R. Maurer F. Eingartner C. Weise K. Weller S. Allogenic cancellous bone graft and a Burch-Schneider ring for acetabular reconstruction in revision hip arthroplasty. J Bone Joint Surg Am. 2001; 83A:862-7.

6. Finkemeir $C G$. Bone-grafting and bone-graft substitutes. J Bone Joint Surg Am. 2002; 84A:454-64.

7. Galia CR. Macedo CAS. Rosito R. Mello TM. Macedo CAS. Uso de enxerto ósseo homólogo e heterólogo em diáfise femoral de ratos: comparação entre enxerto ósseo congelado e liofilizado. Rev Bras Ortop. 2005;40:141-6.

8. Merle AR, Postel M. Funcional results of hip arthroplasty with acrylic prosthesis. J Bone Joint Surg Am. 1954; 36A:351-47.

9. Conn RA, Peterson LFA, Stauffer RN, Ilstrup D. Management of acetabular deficiency: Long-term results of bone grafting the acetabulum in total hip arthroplasty. Orthopaedics Trans. 1985; 9:451-4.
10. De Lee JG, Charnley J. Radiological demarcation of cemented sockets in total hip replacement. Clin Orthop. 1976; 121:20-32.

11. Cohen J. Statistical power analysis for behavioural sciences. London Academic Press. 1969. London. UK.

12. Slooff TJ, Huiskes R, van Horn J, Lemmens AJ. Bone grafting in total hip replacement for acetabular protrusion. Acta Orthop Scand. 1984; 55:593-6.

13. Boldt JG, Dilawari P, Agarwal S, Drabu KJ. Revision total hip arthroplasty using impaction bone grafting with cemented nonpolished stems and charnley cups. J Arthroplasty. 2001;16:94352.

14. Levai JP, Boisgard S. Acetabular reconstruction in total hip revision using a bone graft substitute. Early clinical and radiographic results. Clin Orthop. 1996; 330:08-14.

15. Macedo CAS, Galia CR, Silva ALB, César PC, Sanches PRS, Duarte LS, Muller LM. Comparação da resistência à compressão do osso bovino congelado e liofilizado. Rev Bras Ortop. 1999; 34:529-34.

16. Zasacki W. The efficacy of application of freeze-dried, radiationsterilized bone graft in orthopedic surgery. Clin Orthop. 1991; 272:82-7

17. de Roeck NJ, Drabu KJ. Impaction bone grafting using freezedried allograft in revision hip arthroplasty. J Arthroplasty. 2001; 16:201-6.

18. Thien TM, Welten ML, Verdonschot N, Buma P, Yong P, Schreurs BW. Acetabular revision with impacted freeze-dried cancellous bone chips and a cemented cup: a report of 7 cases at 5 to 9 years' follow-up. J Arthroplasty. 2001; 16:666-70.

19. Charalambides $C$. Beer M, Cobb A. Poor results after augmenting autograft with xenograft (Surgibone) in hip revision surgery. A report of 27 cases. Acta Orthop Scand. 2005; 76:544-9.

20. Donk S, Buma P, Slooff TJ, Gardeniers J, Schreurs BW. Incorporation of morsellised cancelous bone grafts: a study of 24 acetabular biopsy specimes. Clin Orthop. 2002; 396:131-41. 
21. Taylor D. Inactivation of the BSE agent. Comptes Rendus de I'Academie des Scinces Serie III. Sciences de la Vie. 2002; 325:75.

22. Mbithi JN, Springthorpe VS, Sattar AS. Chemical disinfection of hepatitis A virus on environmental surfaces. Appl Environ Microbiol. 1990; 56:3601-4.

23. Aranda-Anzaldo A, Viza D, Busnel RG. Chemical inactivation of human immunodeficiency virus in vitro. J Virol Meth. 1992; 37:7181.

24. Wenz B, Oesch B, Horst M. Analysis of the risk of transmitting bovine spongiform encephalopathy through bone grafts derived from bovine bone. Biomaterials. 2001; 22:1599-606.

25. Cornu O, Bavadekar A, Godts B, Van Tomme J, Delloye C, Banse X (2003). Impaction bone grafting with freeze-dried irradiated bone. Part I. Femoral implant stability: cadaver experiments in a hip simulator. Acta Orthop Scand. 2003; 74: 547-52.

26. Galia CR, Macedo CA, Rosito R, de Mello TM, Camargo LM, Moreira LF. In vitro and in vivo evaluation of lyophilized bovine bone biocompatibility. Clinics. 2008 Dec; 63(6):801-06.
27. Rosito R, Galia CR, Macedo CA, Moreira LF, Quaresma LM, Palma $\mathrm{HM}$. Acetabular reconstruction with human and bovine freezedried bone grafts and a reinforcement device. Clinics. 2008 Aug; 63(4):509-14.

Recebido em 20/09/2008

Aceito para publicação em 15/12/2008

Conflito de interesse: nenhum

Fonte de financiamento: nenhuma

\section{Como citar este artigo:}

Rosito R, Galia CR, Macedo CAS, Quaresma LMAC, Moreira LF. Acompanhamento a médio prazo da reconstrução acetabular com enxerto ósseo liofilizado bovino e dispositivo de reforço. Rev Col Bras Cir. [periódico na Internet] 2009; 36(3). Disponível em URL: http:// www.scielo.br/rcbc

\section{Endereço para correspondência:}

Ricardo Rosito

E-mail: ricardo@rosito.com.br 